\title{
Treatment outcomes and clinical relevance of the Follicular Lymphoma International Prognostic Index in Korean follicular lymphoma patients treated with chemotherapy
}

\author{
Chi Hoon Maeng, ${ }^{1,2}$, Sung Woo $\mathrm{Ahn}^{3}$, Seong-Yoon Ryu', Sungjun $\mathrm{Han}^{3}$, Young Hyeh $\mathrm{Ko}^{4}$, Jun Ho Ji \\ Won Seog Kim ${ }^{2}$, and Seok Jin $\mathrm{Kim}^{2}$
}

\begin{abstract}
${ }^{1}$ Division of Hematology and Oncology, Department of Internal Medicine, Kyung Hee University Medical Center, Seoul; ${ }^{2}$ Division of Hematology and Oncology, Department of Medicine, Departments of ${ }^{3}$ Education and Training and ${ }^{4}$ Pathology, Samsung Medical Center, Sungkyunkwan University School of Medicine, Seoul; ${ }^{5}$ Division of Hematology and Oncology, Department of Internal Medicine, Samsung Changwon Hospital, Sungkyunkwan University School of Medicine, Changwon, Korea
\end{abstract}

Received: July 24, 2014 Revised : December 14, 2014 Accepted: April 15, 2015

\section{Correspondence to \\ Seok Jin Kim, M.D.}

Division of Hematology and Oncology, Department of Medicine, Samsung Medical Center, Sungkyunkwan University School of Medicine, 81 Irwon-ro Gangnam-gu, Seoul o6351, Korea Tel: +82-2-3410-1766 Fax: $+82-2-3410-1754$

Email: kstwoh@skku.edu
Background/Aims: The Follicular Lymphoma International Prognostic Index (FLIPI) and FLIPI2 are well-known prognostic models for patients with follicular lymphoma (FL). However, their prognostic relevance has not been examined before in Korean patients with FL.

Methods: We reviewed clinical and laboratory information from our database of patients between 1995 and 2012. In total, 125 patients were stratified in three categories according to FLIPI or FLIPI2 scores: low-, intermediate-, and high-risk groups. We compared FLIPI and FLIPI2 in terms of progression-free survival (PFS) and overall survival (OS).

Results: Among the 125 patients, the prognostic value of FLIPI and FLIPI2 was evaluated in 73 patients who fulfilled the criteria of both prognostic models. Risk stratification by FLIPI and FLIPI2 showed significant differences in unfavorable parameters among each risk group, particularly between low- and intermediate-risk groups. The high-risk group $\mathrm{b}$ was significantly associated with poor PFS on both FLIPI and FLIPI2 $(p<0.05)$. However, the OS was significantly different only in the risk groups determined by FLIPI2 $(p=0.042)$. In a subgroup analysis of patients who received rituximab-containing chemotherapy, the risk stratification of both prognostic models showed a significant impact on PFS, especially in the low-risk group.

Conclusions: FLIPI and FLIPI2 are appropriate prognostic models in Korean FL patients, especially for discriminating low-risk patients from intermediate- and high-risk groups.

Keywords: Follicular lymphoma; Prognosis; Index

\section{INTRODUCTION}

Follicular lymphoma (FL) is one of the most common subtypes of non-Hodgkin lymphoma (NHL) [1,2]. Howe ever, the incidence of FL is relatively low in Koreans [3], whereas FL accounts for approximately $25 \%$ of lymphoma in Western people [4]. As a result, clinical studies on FL have been performed mainly with Western patients, and information regarding the clinical features and outcomes of FL has been based on Western experiences. 
Since the International Prognostic Index was proposed for aggressive NHL [5], various prognostic models have been developed for each subtype of NHL because clinical behaviors vary according to NHL subtype.

As a prognostic model for FL, the Follicular Lymphoma International Prognostic Index (FLIPI) was first developed in a multinational retrospective study. FLIPI consists of age $>60$ years, Ann Arbor stage III or IV, hemoglobin less than $12 \mathrm{~g} / \mathrm{dL}$, elevated levels of serum lactate dehydrogenase (LDH), and $\geq 5$ involved nodal site areas [6]. However, given that FLIPI was developed according to a retrospective study in the pre-rituximab era, a newer prognostic model, named FLIPI2, was proposed through the analysis of prospectively collected data, including from patients who were treated with rituximab-containing chemotherapy [7]. Thus, age and hemoglobin at diagnosis remain in the FLIPI2, whereas new parameters were added, including elevated serum $\beta_{2}$-microglobulin, largest involved nodes longer than $6 \mathrm{~cm}$ in diameter, and bone marrow (BM) invasion, hemoglobin, and age [7]. Although FLIPI is still used widely, a recent Italian study reported the validation of FLIPI2 and showed that the prognostic value of FLIPI2 was somewhat better than FLIPI [8]. However, the prognostic relevance of neither FLIPI nor FLIPI2 has been validated in Korean patients. Thus, we gathered clinical and laboratory data of FL patients at our institute, and compared their prognosis according to the risk of FLIPI and FLIPI2.

\section{METHODS}

\section{Patients}

We analyzed retrospectively patients who were consecutively diagnosed with lymphoma between 1995 and 2012 at the Samsung Medical Center. Of our patients, 125 were diagnosed pathologically with FL, consistent with the current lymphoma classification at the time of diagnosis, and the histological grades of FL were determined using the World Health Organization (WHO) classification [9]. Demographic findings and clinical variables, including FLIPI and FLIPI2, were collected (Fig. 1). Survival analysis of the population who received any type of chemotherapy was performed. Among them, we finally compared the prognostic relevance of FLIPI

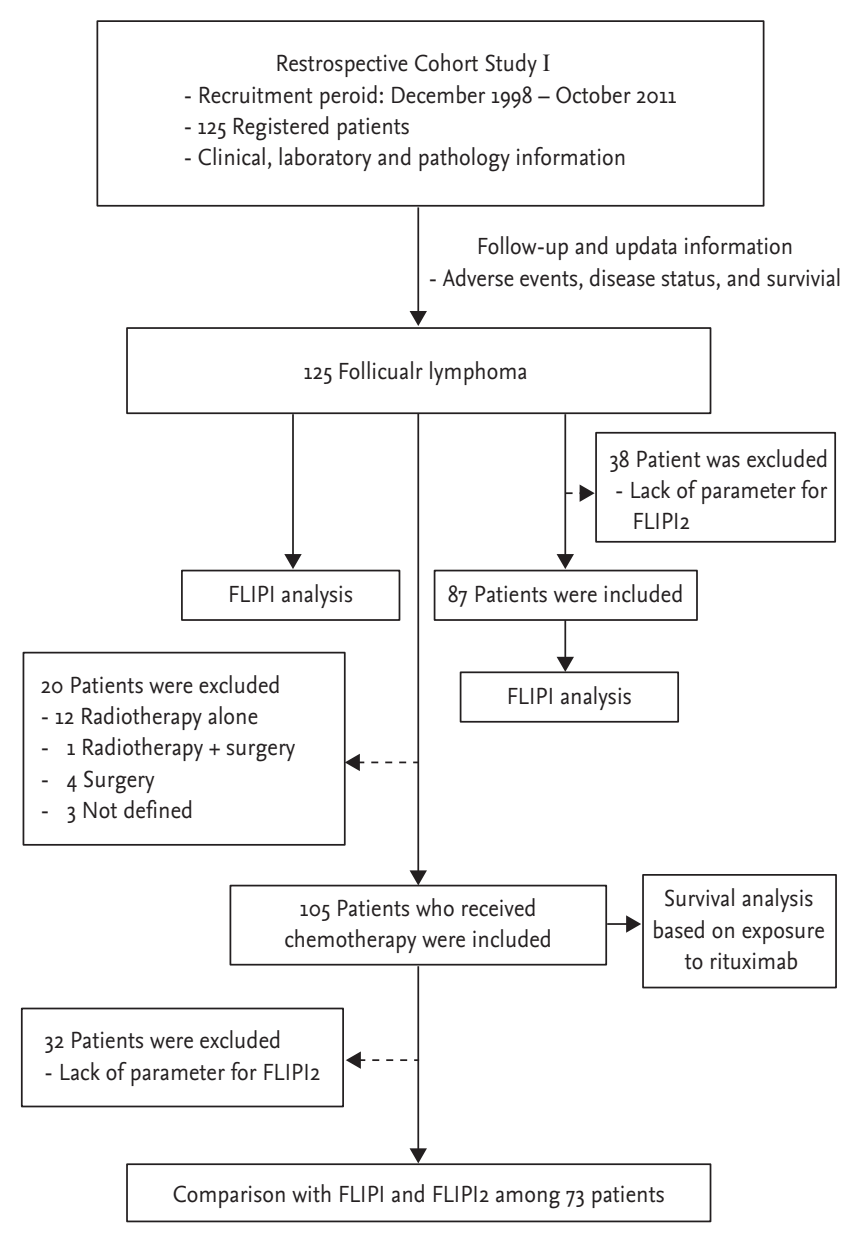

Figure 1. Consolidated Standards of Reporting Trials diagram of cohort. FLIPI, Follicular Lymphoma International Prognostic Index.

and FLIPI2. For a precise comparison, the subset populations were determined according to the following criteria. First, patients should have the clinical and laboratory information that is required for FLIPI and FLIPI2. Thus, patients who were lacking parameters such as serum $\beta_{2}$-microglobulin or imaging data were excluded from the study. Second, patients should receive chemotherapy with curative intent, because the purpose of this study was to explore the impact of risk stratification on the treatment outcome of patients. Thus, patients treated by radiotherapy alone or observed without any kind of treatment were also excluded from this analysis.

\section{Treatment}

As a clinical practice, the chemotherapy regimen was determined basically by treatment guidelines and reim- 
bursement plans of the health insurance system. Thus, the first-line chemotherapy regimens between 1995 and 2006 included cyclophosphamide, vincristine, and prednisone (CVP), cyclophosphamide, doxorubicin, vincristine, and prednisone (CHOP), or other CVP/CHOPlike regimens or chlorambucil because rituximab was not reimbursed by the Korean Health Insurance system at that time. Since rituximab became available for patients with stage III/IV in FL, rituximab has been added, such as R-CVP or R-CHOP. The use of doxorubicin was based basically on the grade of FL. Thus, patients with grade III received CHOP or R-CHOP, whereas patients having grade I or II did not receive doxorubicin. However, doxorubicin was not used for patients who were elderly or frail according to physicians' decisions. When physicians decided that a patient's disease status was aggressive, even though the pathology grade was I or II, CHOP, or R-CHOP was used. Furthermore, rituximab was not reimbursed for stage I/II; patients with grade I/II and stage I/II received CVP. As we focused on the first-line treatment for this analysis, we did not include patients receiving rituximab maintenance therapy.

\section{Statistical analysis}

Progression-free survival (PFS) and overall survival (OS) was analyzed according to the three risk groups of FLIPI and FLIPI2, as reported previously $[6,7]$. Categorical pap rameters were compared among each risk group using chi-square tests. The PFS was calculated from the first date of diagnosis to disease progression, relapse, death from any cause, or the last date of follow-up, whereas the OS was calculated from the first date of diagnosis to death or to the last date of follow-up. Survival curves were estimated by the Kaplan-Meier method and compared using the log-rank test. A two-sided $p<0.05$ was considered to be statistically significant. All analyses were performed using the SPSS version 19.0 (IBM Co., Armonk, NY, USA).

\section{RESULTS}

\section{Patient characteristics}

In total, 125 patients were analyzed; their median age was 53.0 years (range, 16 to 79). Of them, 39 patients were older than 60 years at diagnosis and the median hemo-
Table 1. Patient demographic and baseline clinical features

\begin{tabular}{|c|c|}
\hline Variable & No. (\%) \\
\hline \multicolumn{2}{|l|}{ Age, yr } \\
\hline$\leq 60$ & $86(68.8)$ \\
\hline$>60$ & $39(31.2)$ \\
\hline \multicolumn{2}{|l|}{ Sex } \\
\hline Male & $59(47.2)$ \\
\hline Female & $66(52.8)$ \\
\hline \multicolumn{2}{|c|}{ Hemoglobin, g/dL } \\
\hline$\geq 12$ & $104(83.2)$ \\
\hline$<12$ & $21(16.8)$ \\
\hline \multicolumn{2}{|c|}{ Serum lactate dehydrogenase, IU/L } \\
\hline Normal & $102(81.6)$ \\
\hline Increased & $23(18.4)$ \\
\hline \multicolumn{2}{|c|}{ World Health Organization grade } \\
\hline I & $50(40.0)$ \\
\hline II & $23(18.4)$ \\
\hline III & $46(36.8)$ \\
\hline Unknown & $6(4.8)$ \\
\hline \multicolumn{2}{|l|}{ Ann Arbor stage } \\
\hline I & $32(25.6)$ \\
\hline II & $25(20.0)$ \\
\hline III & $29(23.2)$ \\
\hline IV & $39(31.2)$ \\
\hline \multicolumn{2}{|c|}{ No. of nodal, sites } \\
\hline$<5$ & $90(72.0)$ \\
\hline$\geq 5$ & $34(27.2)$ \\
\hline Unknown & $1(0.8)$ \\
\hline \multicolumn{2}{|c|}{ Serum $\beta_{2}$-microglobulin } \\
\hline$\leq \mathrm{UNL}$ & $67(53.6)$ \\
\hline$>\mathrm{UNL}$ & $31(24.8)$ \\
\hline Unknown & $27(21.6)$ \\
\hline \multicolumn{2}{|c|}{ Longest diameter of lymph node, $\mathrm{cm}$} \\
\hline$\leq 6$ & $104(83.2)$ \\
\hline$>6$ & $6(4.8)$ \\
\hline Unknown & $15(12.0)$ \\
\hline \multicolumn{2}{|c|}{ Bone marrow involvement } \\
\hline$(-)$ & $99(79.2)$ \\
\hline$(+)$ & $26(20.8)$ \\
\hline \multicolumn{2}{|c|}{ Rituximab-containing chemotherapy } \\
\hline$(+)$ & $62(49.6)$ \\
\hline$(-)$ & $63(50.4)$ \\
\hline \multicolumn{2}{|l|}{ FLIPI risk group } \\
\hline Low & $71(56.8)$ \\
\hline Intermediate & $27(21.6)$ \\
\hline High & $27(21.6)$ \\
\hline \multicolumn{2}{|c|}{ FLIPI2 risk group } \\
\hline Low & $36(41.4)$ \\
\hline Intermediate & $39(44.8)$ \\
\hline High & $12(13.8)$ \\
\hline Unknown & 38 (NA) \\
\hline
\end{tabular}

FLIPI, Follicular Lymphoma International Prognostic Index; UNL, upper normal limit; NA, not applicable. 
Table 2. Comparison of clinical characteristics among risk groups based on FLIPI and FLIPI2

\begin{tabular}{|c|c|c|c|c|c|c|c|c|}
\hline \multirow{2}{*}{ Characteristic } & \multicolumn{4}{|c|}{ FLIPI } & \multicolumn{4}{|c|}{ FLIPI2 } \\
\hline & LR & IR & HR & $p$ value & LR & IR & $\mathrm{HR}$ & $p$ value \\
\hline Age, yr & & & & 0.006 & & & & $<0.001$ \\
\hline$\leq 60$ & 31 & 13 & 7 & & 29 & 17 & 5 & \\
\hline$>60$ & 6 & 6 & 10 & & o & 15 & 17 & \\
\hline Bone marrow involvement & & & & $<0.001$ & & & & $<0.001$ \\
\hline$(-)$ & 36 & 10 & 8 & & 29 & 23 & 2 & \\
\hline$(+)$ & 1 & 9 & 9 & & 0 & 9 & 10 & \\
\hline Hemoglobin, g/dL & & & & 0.004 & & & & $<0.001$ \\
\hline$\geq 12$ & 36 & 16 & 11 & & 29 & 30 & 4 & \\
\hline$<12$ & 1 & 3 & 6 & & $\mathrm{O}$ & 2 & 8 & \\
\hline Serum lactate dehydrogenase & & & & $<0.001$ & & & & 0.033 \\
\hline$\leq \mathrm{UNL}$ & 36 & 19 & 7 & & 28 & 26 & 8 & \\
\hline$>$ UNL & 1 & 0 & 10 & & 1 & 6 & 4 & \\
\hline Ann Arbor stage & & & & $<0.001$ & & & & $<0.001$ \\
\hline I-II & 24 & 1 & o & & 17 & 8 & o & \\
\hline III-IV & 13 & 18 & 17 & & 12 & 24 & 12 & \\
\hline Rituximab-containing chemotherapy & & & & 0.010 & & & & 0.268 \\
\hline$(+)$ & 20 & 17 & 14 & & 17 & 15 & 9 & \\
\hline$(-)$ & 17 & 2 & 3 & & 12 & 7 & 3 & \\
\hline No. of nodal sites & & & & $<0.001$ & & & & 0.021 \\
\hline$<5$ & 36 & 8 & 2 & & 24 & 17 & 5 & \\
\hline$\geq 5$ & 1 & 11 & 14 & & 5 & 15 & 6 & \\
\hline$\beta_{2}$-Microglobulin & & & & $<0.001$ & & & & $<0.001$ \\
\hline$\leq \mathrm{UNL}$ & 32 & 11 & 6 & & 29 & 18 & 2 & \\
\hline > UNL & 5 & 8 & 11 & & o & 14 & 10 & \\
\hline Lymph node size, $\mathrm{cm}$ & & & & 0.018 & & & & 0.013 \\
\hline$\leq 6$ & 37 & 18 & 14 & & 29 & 31 & 9 & \\
\hline$>6$ & 0 & 1 & 3 & & 0 & 1 & 3 & \\
\hline World Health Organization histology grade & & & & 0.027 & & & & 0.009 \\
\hline $\mathrm{I} / \mathrm{II}$ & 16 & 15 & 14 & & 12 & 24 & 9 & \\
\hline III & 19 & 3 & 3 & & 15 & 7 & 3 & \\
\hline
\end{tabular}

FLIPI, Follicular Lymphoma International Prognostic Index; LR, low risk; IR, intermediate risk; HR, high risk; UNL, upper normal limit.

globin level at diagnosis was $13.4 \mathrm{~g} / \mathrm{dL}$ (range, 8.6 to 17.8) (Table 1). Of all the patients, $58.4 \%$ presented with WHO histological grade I or II FL; however, grade was unknown in six patients due to lack of available data (Table 1). The median follow-up duration of patients was 4.4 years (range, 1.3 to 13.9). R-CVP was the most commonly used regimen $(n=35)$, followed by CHOP $(n=15)$ and $\mathrm{R}-\mathrm{CHOP}(\mathrm{n}=14)$. Thus, among 66 patients with stage III/
IV, except for two patients without information about FL grade, 17 patients with grade III received CHOP ( $\mathrm{n}=$ 8) or R-CHOP $(n=9)$ whereas 49 patients with grade I/ II received R-CVP $(\mathrm{n}=35)$, R-CHOP $(\mathrm{n}=5)$, CHOP $(\mathrm{n}=$ 7), or CVP $(n=2)$. Other patients with stage I/II received CVP or CVP-like regimens or chlorambucil, according to our treatment practice. Also, 20 patients with stage I/ II were not initially treated with chemotherapy. Instead, 
Table 3. PFS and OS based on FLIPI and FLIPI2

\begin{tabular}{|c|c|c|c|c|}
\hline \multirow{2}{*}{ Variable } & \multicolumn{2}{|c|}{ Progression-free survival, \% } & \multicolumn{2}{|c|}{ Overall survival, \% } \\
\hline & 3-Year & 5-Year & 3-Year & 5-Year \\
\hline \multicolumn{5}{|l|}{ FLIPI } \\
\hline Low & 91.9 & 91.9 & 100.0 & 94.7 \\
\hline Intermediate & 66.8 & 50.1 & 94.1 & 70.6 \\
\hline High & $57 \cdot 0$ & $57 \cdot 0$ & 81.9 & 81.9 \\
\hline \multicolumn{5}{|l|}{ FLIPI2 } \\
\hline Low & 96.3 & 96.3 & 100.0 & 94.7 \\
\hline Intermediate & 69.2 & 61.5 & 94.1 & 70.6 \\
\hline High & 64.2 & 64.2 & 81.9 & 81.9 \\
\hline
\end{tabular}

FLIPI, Follicular Lymphoma International Prognostic Index.

Table 4. Univariate analysis of survival outcomes based on variables of FILIPI and FLIPI2

\begin{tabular}{lcc}
\hline Variable & \multicolumn{2}{c}{$p$ value } \\
\hline Age $>60$ yr & Progression-free survival & Overall survival \\
\cline { 2 - 3 } Hemoglobin $<12$ g/dL & 0.262 & 0.002 \\
Serum lactate dehydrogenase (increased), IU/L & 0.283 & 0.450 \\
Ann Arbor stage (III/IV) & 0.248 & 0.972 \\
Lymph node involvement $(\geq 5$ sites) & 0.023 & 0.126 \\
Serum $\beta_{2}$-microglobulin $(>$ upper normal limits) & 0.030 & 0.362 \\
Longest diameter of lymph node $(>6 \mathrm{~cm})$ & $<0.001$ & 0.002 \\
Bone marrow involvement (positive) & 0.213 & 0.305 \\
\hline
\end{tabular}

FLIPI, Follicular Lymphoma International Prognostic Index.

they were treated with surgery $(n=5)$ or radiotherapy $(\mathrm{n}=12)$. The remaining three asymptomatic patients with low tumor burden received no treatment and were monitored closely.

\section{Risk stratification based on FLIPI and FLIPI2}

According to the risk model of FLIPI, more than a half of the patients $(\mathrm{n}=71,56.8 \%)$ belonged to the low-risk group, whereas the intermediate- and high-risk groups accounted for $43.2 \%$ of patients (Table 1). Regarding FLIPI2, 38 patients could not be classified due to lack of clinical data. We defined the target population as those treated with chemotherapy and with available clinical parameters for FLIPI and FLIPI2. We compared clinical variables of patients between the three risk groups according to FLIPI and FLIPI2 (Table 2, Fig. 1). The risk groups of FLIPI were associated significantly with five unfavorable clinical parameters, BM involvement, stage, rituximab-containing chemotherapy, number of nodal sites, and level of $\beta_{2}$-microglobulin, especially the lowand high-risk groups. However, the comparison of intermediate- and high-risk FLIPI groups did not show any significant differences among the variables except for serum LDH. Risk stratification by FLIPI2 showed a similar pattern to the FLIPI score. Thus, the low-risk group of FLIPI2 showed a significant difference from the intermediate- and high-risk groups in terms of unfavorable parameters, such as age, BM involvement, and stage (Supplementary Table 1). When the WHO grades were compared according to the risk of FLIPI and FLIPI2, the majority of patients with grade III belonged to the lowrisk group rather than the intermediate- or high-risk group of FLIPI and FLIPI2 $(p=0.027$ and 0.009 , respectively). Thus, there was no tendency for high grade being in the high-risk group of FLIPI or FLIPI2 (Table 2). 

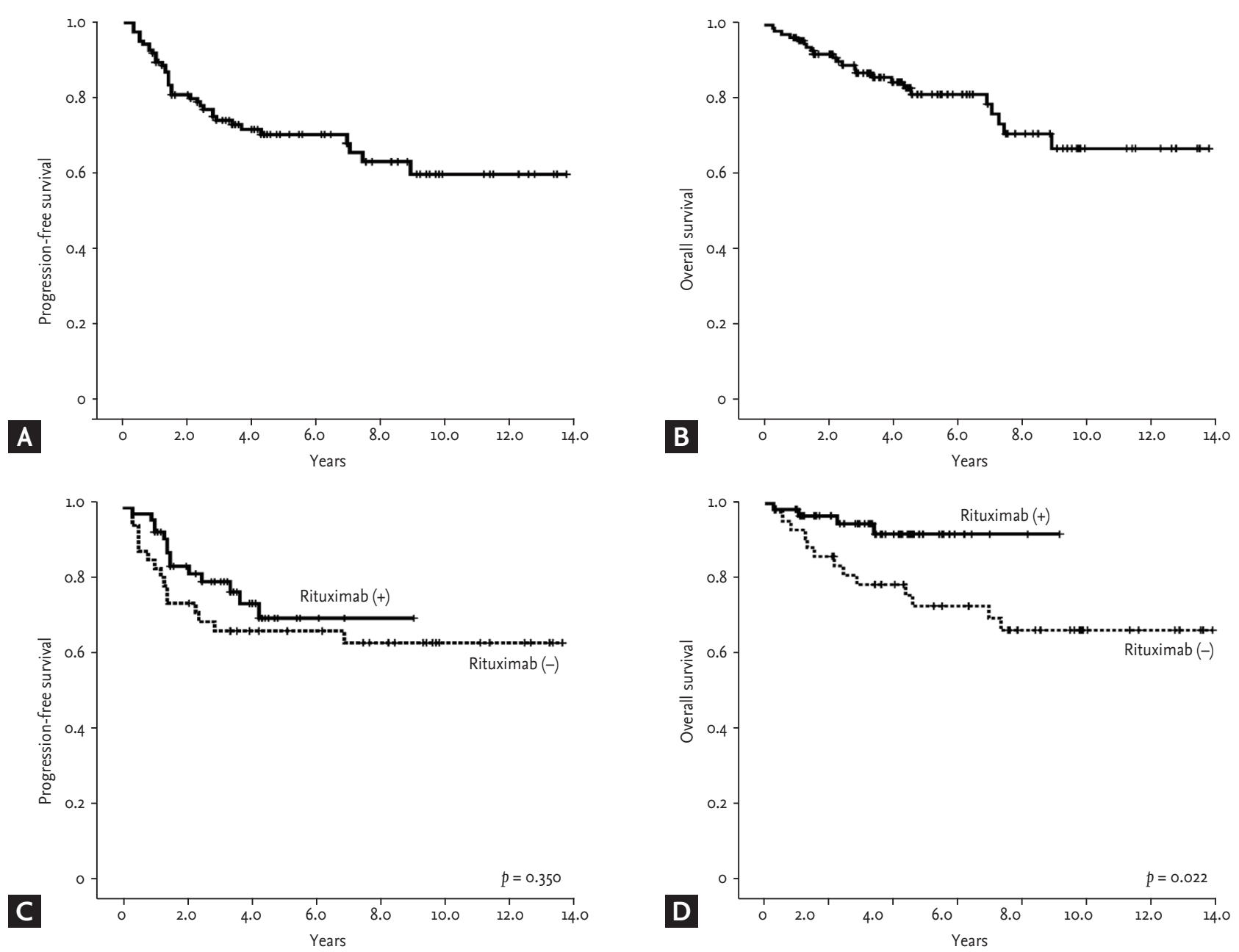

Figure 2. Progression-free survival and overall survival of (A, B) the entire follicular lymphoma cohort and (C, D) chemotherapytreated patients (rituximab).

\section{Survival analysis}

Neither the median PFS nor the median OS of the 125 patients was reached; this might have been because asymptomatic patients with an indolent clinical course were also included (Fig. $2 \mathrm{~A}$ and $2 \mathrm{~B}$ ). When OS and PFS were compared among patients who received chemotherapy ( $n=105$ ) according to the use of rituximab, only the OS of patients receiving rituximab-containing chemotherapy was significantly better than patients without rituximab (Fig. $2 \mathrm{C}$ and $2 \mathrm{D}$ ). Risk stratification by both FLIPI and FLIPI2 scores showed a significant difference in PFS among the three risk groups (Fig. 3A and 3B). In particular, the low-risk group, defined by FLIPI or FLIPI2, was strongly associated with better PFS than the intermediate- and high-risk groups. However, there was no significant difference in PFS between the inter- mediate- and high-risk groups. The risk groups of FLIPI2 also showed a significant association with OS, but FLIPI did not (Fig. ${ }_{3} \mathrm{C}$ and ${ }_{3} \mathrm{D}$ ). The comparison of PFS in patients receiving rituximab-containing chemotherapy showed a significant association of PFS with FLIPI and FLIPI2 (Fig. 4A and 4B). However, OS was not significantly discriminated by either index (Fig. $4 \mathrm{C}$ and $4 \mathrm{D}$ ).

\section{Comparison of parameters of FLIPI and FLIPI/2}

FLIPI and FLIPI2 showed a significant association with PFS regardless of the use of rituximab. However, the low-risk group showed superior PFS to the intermediate- and high- risk groups whereas the difference between the intermediate- and high-risk groups was not significant. Thus, 3- and 5-year PFS and OS, based on FLIPI and FLIPI2, were significantly different between 

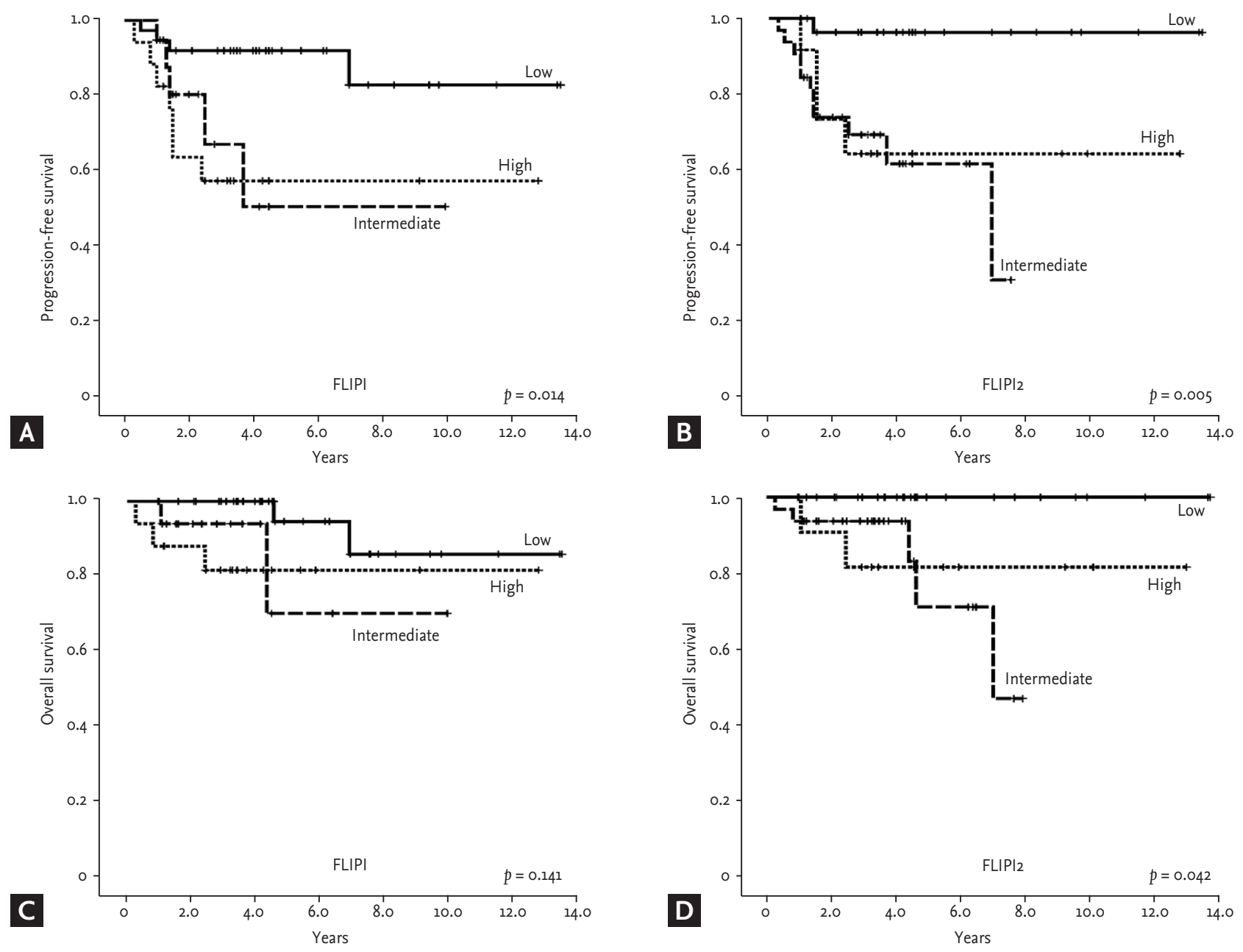

Figure 3. (A, B) Progression-free survival and (C, D) overall survival among subset populations based on FLIPI and FLIPI2 risk groups. FLIPI, Follicular Lymphoma International Prognostic Index.

the low- and intermediate-risk groups (Table 3). When we performed univariate analysis with each parameter of FLIPI and FLIPI2 for OS and PFS, Ann Arbor stage, number of lymph nodes, and $\beta_{2}$-microglobulin were significantly associated with PFS (Table 4). Although age was not statistically responsible for PFS, it was significantly related to OS, together with $\beta_{2}$-microglobulin. Thus, $\beta_{2}$-microglobulin was independently prognostic for OS and PFS in the multivariate analysis.

\section{DISCUSSION}

This is the first reported study to investigate the clinical relevance of two prognostic models, FLIPI and FLIPI2, in Korean patients with FL. The clinical and laboratory characteristics of patients at diagnosis were comparable to those in previous studies. However, the proportions of patients with BM involvement and stage III/IV (26\% and $62 \%$, respectively) (Table 1 ) were relatively low because $40 \%$ to $60 \%$ of patients with FL showed BM involvement, and more than $70 \%$ of patients had stage III or IV in previous studies $[6,10,11]$. Although the reason was not clear, it might be related to the retrospective nature of this study and that the study population was from a single institution. In a nationwide survey, the proportion of stage III/IV might differ from our results. In addition, the number of patients in the high-risk group on FLIPI2 was relatively lower than that on FLIPI in our study. This low proportion of the high-risk group on FLIPI2 (12\%) compared with other reports is thought to result from the exclusion of a substantial number of 

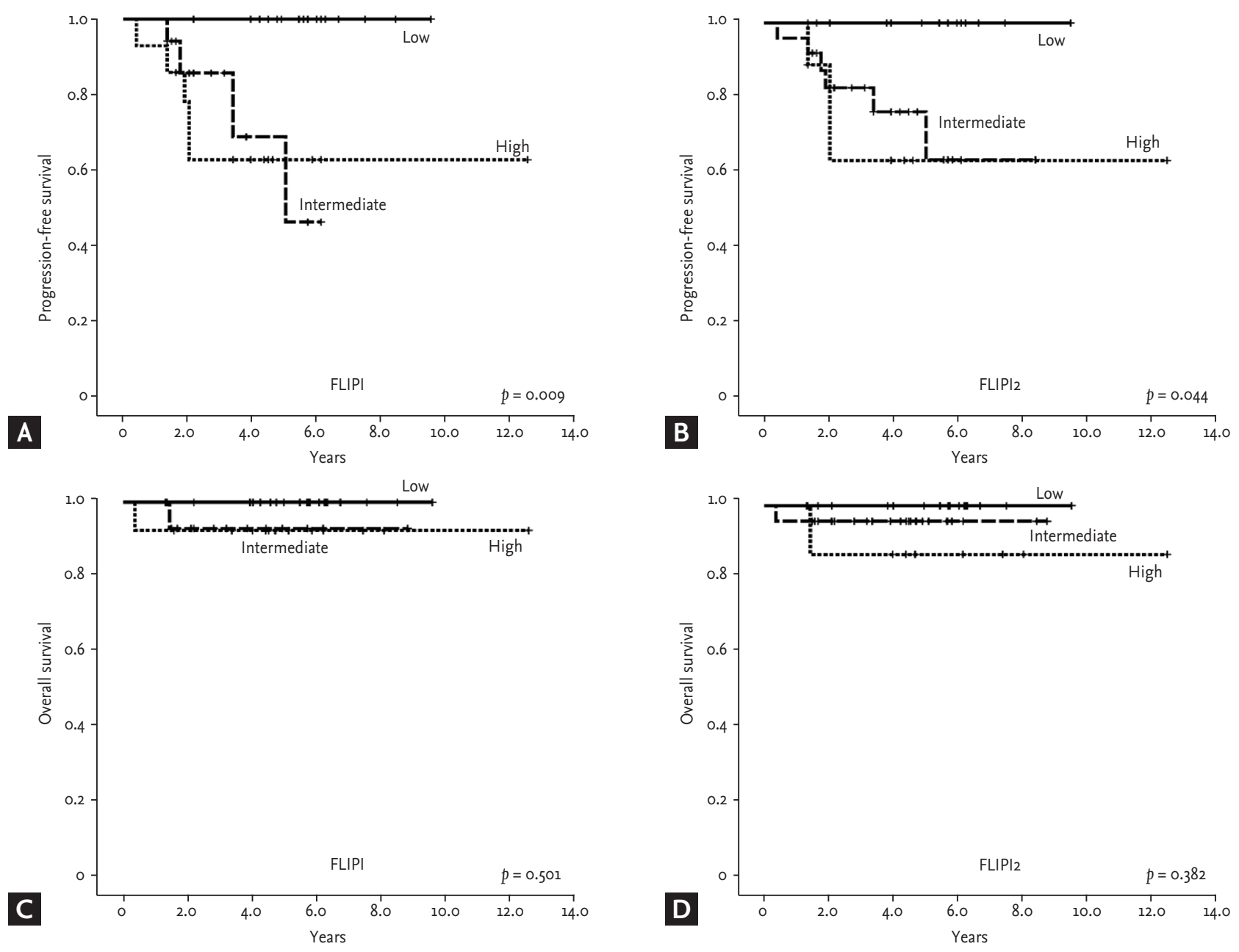

Figure 4. (A, B) Progression-free survival and (C, D) overall survival among patients treated by rituximab-containing chemotherapy based on FLIPI and FLIPI2 risk groups. FLIPI, Follicular Lymphoma International Prognostic Index.

patients $(\mathrm{n}=38)$ when FLIPI2 scores were calculated due to missing data. Thus, further study is warranted to address the prognostic roles of FLIPI and FLIPI2 with a larger study population.

Although 'watch and wait' has been an important treatment strategy for indolent lymphomas, including FL, the need for systemic chemotherapy is growing because patients often show rapidly progressing symptomatic disease from an asymptomatic state. Furthermore, the improved efficacy of chemotherapy due to the introduction of new drugs has contributed to more use of chemotherapy than in the past. For example, rituximab, an anti-CD20 monoclonal antibody, has shown a significant impact on the outcome of patients with FL since it was introduced for the treatment of B-cell lymphomas in Korea. Thus, as a part of combination che- motherapy regimens, such as R-CVP or R-CHOP, rituximab has been used widely, and treatment outcomes have improved versus chemotherapy alone. Our study also showed better outcomes for patients receiving rituximab-containing chemotherapy compared with the pre-rituximab era. The 5-year OS of the high-risk group in our study (81.9\%) was superior to that of the first report introducing FLIPI (52.5\%) [6]. However, our study showed no significant difference in PFS according to the use of rituximab-containing chemotherapy, unlike previous studies [12]. This might be related to the uneven distribution of subsets of each risk group and the patients who received rituximab-containing chemotherapy. Based on FLIPI, there was a tendency toward the high-risk group having more patients receiving rituximab-containing chemotherapy. Similarly, the number 
of patients with low risk was more than in the intermediate plus high-risk groups among those who did not receive rituximab. As a result, the potential benefit of rituximab might be offset by a higher risk of disease.

Although FLIPI and FLIPI2 are commonly used prognostic indexes in clinical practice at present because of their simplicity, several studies have challenged their prognostic value for the management of patients with FL [13-17]. In particular, survival outcome in the intermediate-risk group failed to show a statistically significant difference from the high-risk group [13]. A recent retrospective study with Japanese patients also failed to show a significant association of FLIPI with prognosis in patients with FL [17]. In our study, FLIPI and FLIPI2 were likely to discriminate between each risk group. Especially, the prognostic value for the low-risk group appeared to be stronger than that for the intermediateand high-risk groups. However, the 5-year PFS of the intermediate-risk group of FLIPI2 (61.5\%) did not differ from that of the high-risk group (64.2\%), in contrast to a previous study showing 5-year PFS rates of $51.2 \%$ and $18.8 \%$ in the intermediate- and high-risk groups, respectively, on FLIPI2 [7]. This might be related to improved survival outcomes in the high-risk group.

Our study showed similar patterns of risk stratification with both FLIPI and FLIPI2, and similar associations with PFS, particularly in the low-risk group. However, this study had a limitation with respect to the small number of patients available for analyzing FLIPI2. As a result, only 12 patients were classified in the high-risk group by FLIPI2, and this small number of patients might limit the statistical power. When the prognostic value of each parameter of FLIPI and FLIPI2 was analyzed, serum $\beta_{2}$-microglobulin was found to be an independent prognostic factor for PFS and OS. These results might suggest a role of serum $\beta_{2}$-microglobulin as a single prognostic factor for FL; this should be confirmed by a larger population-based prospective study.

In conclusion, FLIPI and FLIPI2 are valuable for discriminating Korean patients, especially with regard to low-, intermediate- and high-risk groups. Given the limitations related to the retrospective nature of this study, our results should be confirmed in a larger prospective study.

\section{KEY MESSAGE}

1. Follicular Lymphoma International Prognostic Index (FLIPI) and FLIPI2 are feasible prognostic models in Korean follicular lymphoma patients.

2. These models can identify low-risk patients better compared with intermediate or high-risk patients, especially in terms of progression-free survival.

3. Discrimination between intermediate- and high-risk patients based on FLIPI or FLIPI2 was shown to be somewhat ambiguous.

\section{Conflict of interest}

No potential conflict of interest relevant to this article was reported.

\section{REFERENCES}

1. Morton LM, Wang SS, Devesa SS, Hartge P, Weisenburger DD, Linet MS. Lymphoma incidence patterns by WHO subtype in the United States, 1992-2001. Blood 2006;107:265-276.

2. Huh J. Epidemiologic overview of malignant lymphoma. Korean J Hematol 2012;47:92-104.

3. Park HJ, Park EH, Jung KW, et al. Statistics of hematologic malignancies in Korea: incidence, prevalence and survival rates from 1999 to 2008. Korean J Hematol 2012;47:28-38.

4. Tan D, Horning SJ. Follicular lymphoma: clinical features and treatment. Hematol Oncol Clin North Am 2008;22:863-882.

5. The International Non-Hodgkin's Lymphoma Prognostic Factors Project. A predictive model for aggressive non-Hodgkin's lymphoma. N Engl J Med 1993;329:987994.

6. Solal-Celigny P, Roy P, Colombat P, et al. Follicular lymphoma international prognostic index. Blood 2004;104:12581265.

7. Federico M, Bellei M, Marcheselli L, et al. Follicular lymphoma international prognostic index 2: a new prognostic index for follicular lymphoma developed by the international follicular lymphoma prognostic factor project. J Clin Oncol 2009;27:4555-4562. 
8. Arcaini L, Merli M, Passamonti F, et al. Validation of follicular lymphoma international prognostic index 2 (FLIPI2) score in an independent series of follicular lymphoma patients. Br J Haematol 2010;149:455-457.

9. Swerdlow SH CE, Harris NL, et al. World Health Organization Classification of Tumours of Haematopoietic and Lymphoid Tissues. Lyon: IARC Press, 2008.

10. The Non-Hodgkin's Lymphoma Classification Project. A clinical evaluation of the International Lymphoma Study Group classification of non-Hodgkin's lymphoma. Blood 1997;89:3909-3918.

11. Canioni D, Brice P, Lepage E, et al. Bone marrow histological patterns can predict survival of patients with grade 1 or 2 follicular lymphoma: a study from the Groupe d'Etude des Lymphomes Folliculaires. Br J Haematol 2004;126:364-371.

12. Pettengell R. Advanced-stage follicular lymphoma in the rituximab era: when should patients receive anthracycline-based chemotherapy? Drugs 2009;69:1727-1737.

13. Formica V, Norman AR, Cunningham D, Wotherspoon A,
Oates J, Chong G. Utility of the Follicular Lymphoma International Prognostic Index and the International Prognostic Index in assessing prognosis and predicting firstline treatment efficacy in follicular lymphoma patients. Acta Haematol 2009;122:193-199.

14. Luminari S, Cox MC, Montanini A, Federico M. Prognostic tools in follicular lymphomas. Expert Rev Hematol 2009;2:549-562.

15. Solal-Celigny P, Cahu X, Cartron G. Follicular lymphoma prognostic factors in the modern era: what is clinically meaningful? Int J Hematol 2010;92:246-254.

16. Van de Schans SA, Steyerberg EW, Nijziel MR, Creemers GJ, Janssen-Heijnen ML, van Spronsen DJ. Validation, revision and extension of the Follicular LymphomaInternational Prognostic Index (FLIPI) in a population-based setting. Ann Oncol 2009;20:1697-1702.

17. Numata A, Tomita N, Fujimaki K, et al. Retrospective study of the utility of FLIPI/FLIPI-2 for follicular lymphoma patients treated with R-CHOP. J Clin Exp Hematop 2012;52:77-79. 


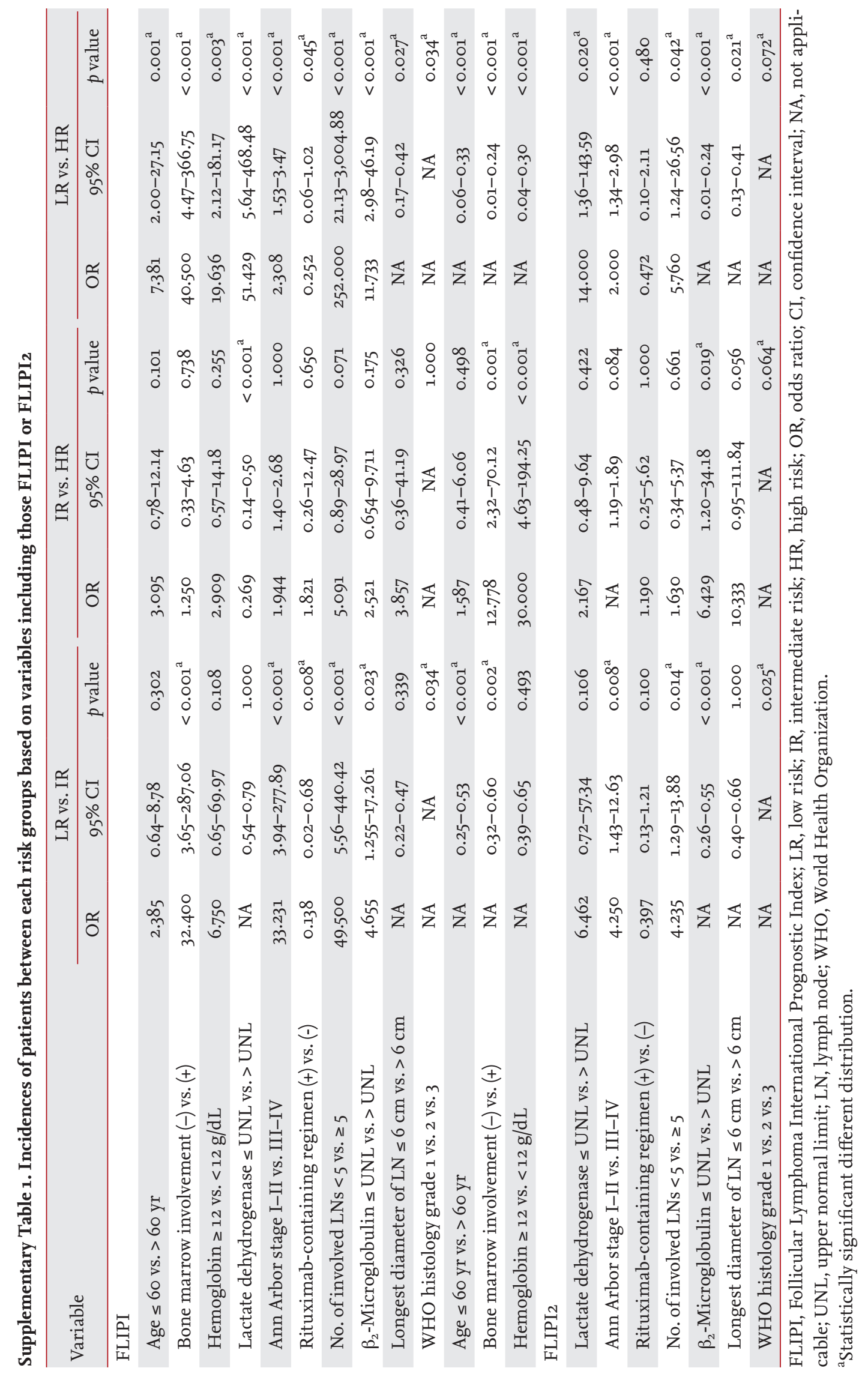

